\title{
RELATION BETWEEN HELICAL ANISOTROPY AND ASYMMETRIC GMI EFFECT
}

\author{
Jozef. KRAVČÁK \\ Department of Physics, Faculty of Electrical Engineering and Informatics, \\ Technical University of Košice, Park Komenského 2, 04200 Košice, Slovak Republic, tel. +421 55602 2601, \\ e-mail: jozef.kravcak@tuke.sk
}

\begin{abstract}
The giant magnetoimpedance (GMI) dependence on the external magnetic field strength $\mathrm{H}$ in as cast $\mathrm{Co}_{70.5} \mathrm{Fe}_{4.5} \mathrm{Si}_{15} \mathrm{~B}_{10}$ amorphous microwire exhibits double-peak behaviour. The position of sharp peaks is symmetrical with respect to zero external magnetic fields strength $H=0$ and corresponds to the critical field strength $H= \pm H_{m}$. The maximum $G M I$ ratio $(\Delta \mathrm{Z} / \mathrm{Z})_{\max }$ dependence on the amplitude $i_{a c}$ is analysed by means of the helical anisotropy $\left(0<\alpha<90^{\circ}\right)$. The additional dc bias current $i_{d c}$ in the interval from 0 to $0.25 \mathrm{~mA}$ with $i_{a c}=0.1 \mathrm{~mA}$ was applied in order to observe the asymmetric GMI effect. The hysteresis in asymmetric GMI(H) dependences is explained by an irreversible magnetization rotation.
\end{abstract}

Keywords: amorphous microwire, circular domain, circular domain wall, GMI effect, helical anisotropy

\section{INTRODUCTION}

The scanning electron microscopy (SEM) image (Fig. 1) displays an end of a as cast glass-coated ferromagnetic amorphous thin $\mathrm{Co}_{70.5} \mathrm{Fe}_{4.5} \mathrm{Si}_{15} \mathrm{~B}_{10}$ wire (microwire) of a diameter of $17.8 \mu \mathrm{m}$ prepared by TaylorUlitovski technique [1]. During this preparation procedure the radial, axial and torsion mechanical stresses are induced in the metallic central part of the microwire (bright in Fig. 1) of a diameter $8.1 \mu \mathrm{m}$. The preferential orientation of the spontaneous magnetization (magnetic anisotropy) in the microwire is given by magnetostriction and shape anisotropy. In cobalt based microwires we observe a relatively small negative magnetostriction, which results in the creation of a wide almost circularly magnetized shell domain structure and a narrow axially magnetized core [2]. Different mechanical properties of the ferromagnetic metallic central part and of the glass cover (dark gray in Fig. 1) of the microwire are responsible for slight deviation of spontaneous magnetization from circular direction in the shell of the microwire (helical magnetic anisotropy). The giant magnetoimpedance (GMI) effect, which is mainly a surface effect, is very sensitive to the rotation of magnetization in the shell of the microwire [3]. For this reason a measurement of GMI effect is often used to determine the surface magnetic properties of cobalt based microwires.

\section{THEORETICAL DESCRIPTION}

In case of a circular wire it is convenient to use cylindrical coordinates. Helical magnetic anisotropy is given by a preferential orientation (easy axis) of the spontaneous magnetization $\boldsymbol{M}_{\mathbf{0}}$ of the microwire at zero external magnetic field what can be expressed as $\boldsymbol{M}_{\mathbf{0}}=(0$, $\left.M_{\phi}, M_{\mathrm{z}}\right)=\left(0, M_{\mathrm{s}} \cos \alpha, M_{\mathrm{s}} \sin \alpha\right)$, where $M_{\mathrm{s}}$ is the saturation magnetization, $\alpha$ is the angle of deviation of the easy axis of magnetization from the circular direction of the microwire. The value of $\alpha$ determines the shape of the magnetization curve of the microwire during its circular magnetization, see Fig. 2, where $K_{u}$ is a constant of the helical magnetic anisotropy [4]. The magnetization curve for $\alpha=90^{\circ}$ represents a hard axis of magnetization along the longitudinal wire $z$-axis. The classical quasistatic theory of the skin effect gives the expression for the impedance of a cylindrical conductor with radius $a$ :

$$
\frac{Z}{R_{d c}}=\frac{k a}{2} \frac{J_{0}(k a)}{J_{1}(k a)},
$$

where $k=(1+\mathrm{i}) / \delta, \delta=\sqrt{2 \rho / \omega \mu_{\varphi}}, J_{i}$ denotes the Bessel function of the $i$-th order, $\rho$ is resistivity, $\omega$ is angular frequency and $\mu_{\phi}$ is circular permeability [3]. The quasistatic model based on the minimization of the free energy of the domain structure was developed [5]. This model is successful in explaining the existence of peaks in the GMI dependence in Fig. 3, if only a reversible magnetization rotation is present, Fig. $2\left(\alpha=90^{\circ}\right)$.

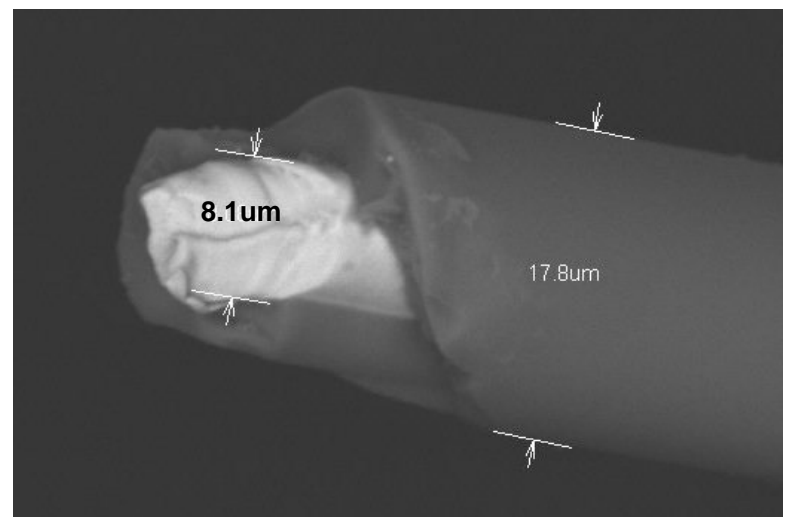

Fig. 1 SEM image of glass-coated ferromagnetic amorphous CoFeSiB microwire

\section{EXPERIMENTAL RESULTS}

The GMI ratio is usually defined as $\Delta Z / Z=\{|Z(H)|-$ $\left.\left|Z\left(H_{\max }\right)\right|\right\} /\left|Z\left(H_{\max }\right)\right|$, where $|Z|$ is the impedance modulus and $H_{\max }$ is the maximum of the measuring static axial field $H$, at which the sample is considered to be magnetically saturated along the $z$-axis. In the presented experiments the GMI ratio at the frequency of $1 \mathrm{MHz}$ was 
measured at different amplitudes $i_{\mathrm{ac}}$ of the harmonic current flowing through the as cast $\mathrm{Co}_{70.5} \mathrm{Fe}_{4.5} \mathrm{Si}_{15} \mathrm{~B}_{10}$ microwire [6]. From $\mathrm{GMI}(H)$ ratio dependences, which exhibit a double-peak behaviour as in Fig. 3, the maximum value of GMI ratio $(\Delta Z / Z)_{\max }$ and its positions $H_{\mathrm{m}}$ and $+H_{\mathrm{m}}$ were plotted as functions of amplitudes $i_{\mathrm{ac}}$ in Fig. 4. The application of an additional dc bias current $i_{\mathrm{dc}}$ in the interval $0-0.25 \mathrm{~mA}$ altogether with $i_{\mathrm{ac}}=0.1 \mathrm{~mA}$ revealed an asymmetric character of the $\operatorname{GMI}(H)$ dependences displayed in Fig. 5.

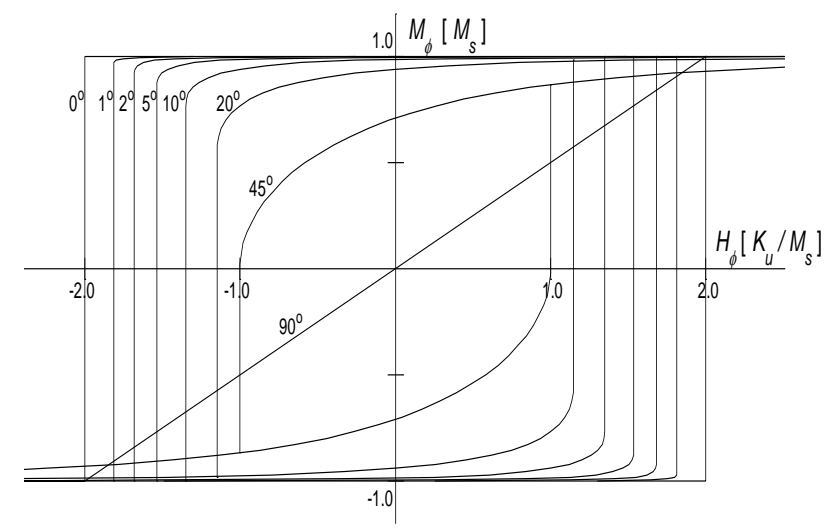

Fig. 2 Calculated reduced magnetization curves $M_{\phi}\left(H_{\phi}\right)$ in case of irreversible magnetization rotation and helical magnetic anisotropy for various value of $\alpha$ (in degrees)

\section{DISCUSSION}

The measured $\operatorname{GMI}(H)$ dependence in Fig. 3 displays the double-peak behaviour. The positions of the couple of sharp peaks are symmetrical with respect to zero external magnetic fields strength $H=0$ and correspond to the critical field strengths $H= \pm H_{m}$. Possible explanation is that for very low amplitudes $i_{\text {ac }}$ (or circular field strength $H_{\phi}=$ $\left.i_{\mathrm{ac}} / 2 \pi a\right)$ a reversible magnetization rotation only takes place in the shell of the microwire, if it is taken into account that a reversible domain wall motion at the frequency of $1 \mathrm{MHz}$ is negligible due to strong damping process [7].

The obtained $(\Delta Z / Z)_{\max }$ dependence on $i_{\mathrm{ac}}$ in Fig. 4 exhibits maximum at amplitude $i_{\mathrm{ac}}=1 \mathrm{~mA}$ (or $H_{\phi}=22.7 \mathrm{Am}^{-1}$ ), which indicates the limit for the reversible magnetization rotation. Onset of an irreversible magnetization rotational process causes the decrease of $(\Delta Z / Z)_{\max }$ at amplitudes $i_{\mathrm{ac}}>1 \mathrm{~mA}$ (or $H_{\phi}>22.7 \mathrm{Am}^{-1}$ ). We also observe a hysteretic behaviour in the measured GMI dependences in Figs. 3,5. The irreversible rotation of magnetization can be explained by the helical magnetic anisotropy $\left(0<\alpha<90^{\circ}\right)$, where the rotational jumps of magnetization into the opposite direction occur. The magnitude of rotational jumps of magnetization depends on the angle $\alpha$ as well as on the amplitude $H_{\phi}=i_{\mathrm{ac}} / 2 \pi a$, see Fig. 2. Onset of an irreversible magnetization rotational process also causes the decrease of critical field strength $H= \pm H_{m}$ in Fig. 4 (down), which corresponds to the decrease of critical field strength in calculated magnetization curves displayed in Fig. 2.

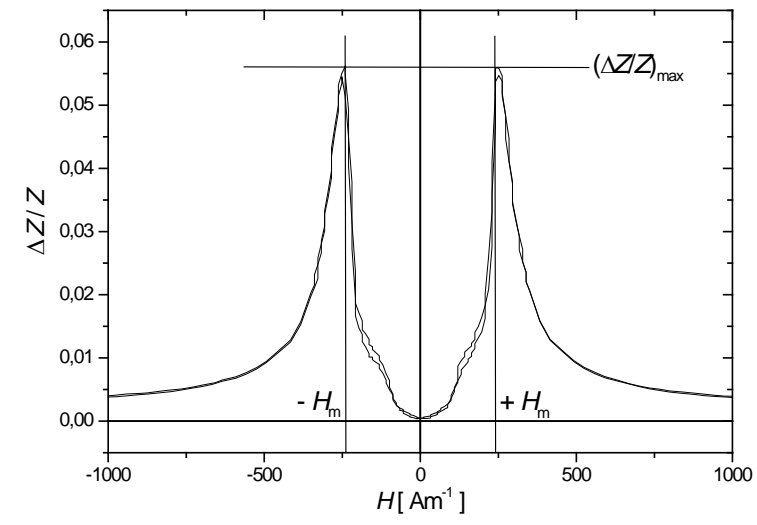

Fig. 3 GMI dependence in $\mathrm{Co}_{70.5} \mathrm{Fe}_{4.5} \mathrm{Si}_{15} \mathrm{~B}_{10}$ microwire measured at the frequency $1 \mathrm{MHz}$ and at the amplitude $\mathrm{i}_{\mathrm{ac}}=1 \mathrm{~mA}$
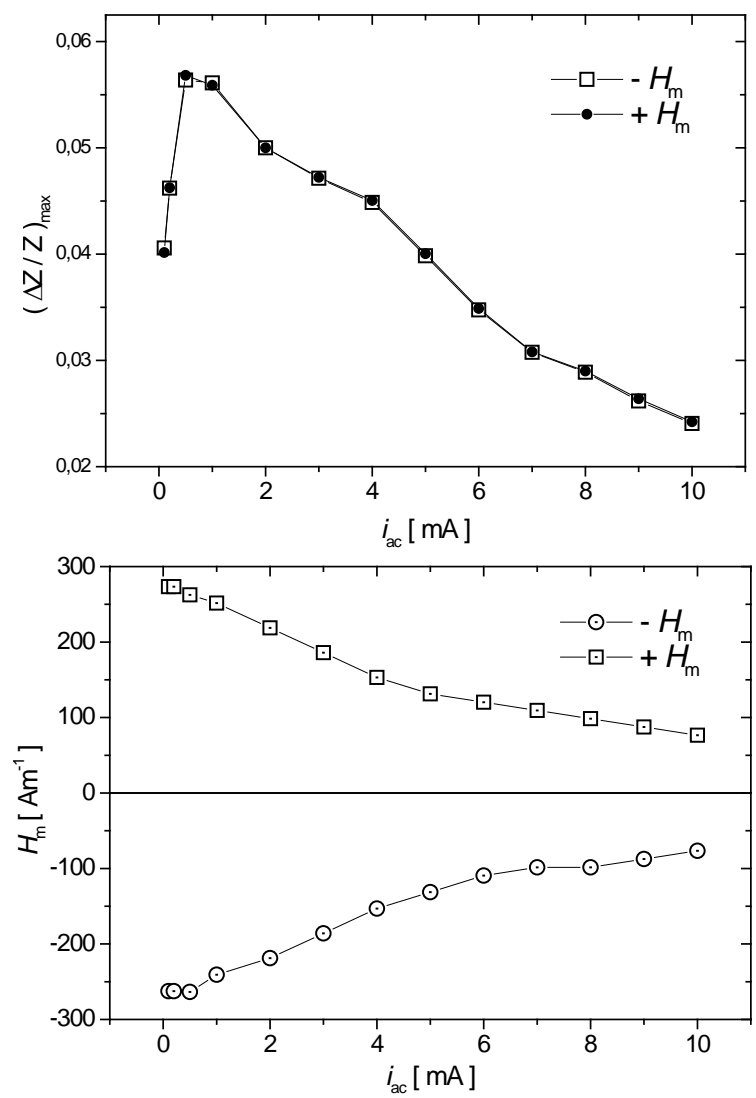

Fig. 4 Maximum value of GMI ratio $(\Delta Z / Z)_{\max }$ (up) and its positions $-H_{\mathrm{m}}$ and $+H_{\mathrm{m}}$ (down) as function of amplitude $i_{\mathrm{ac}}$ determined from GMI dependences in $\mathrm{Co}_{70.5} \mathrm{Fe}_{4.5} \mathrm{Si}_{15} \mathrm{~B}_{10}$ microwire measured at the frequency of $1 \mathrm{MHz}$, as in Fig. 3

When the additional dc bias current $i_{\mathrm{dc}}$ in the interval 0-0.25 mA with $i_{\mathrm{ac}}=0.1 \mathrm{~mA}$ was applied, the asymmetric GMI effect was observed (Fig. 5). The small $i_{\mathrm{dc}}<0.1 \mathrm{~mA}$ diverts the magnetization vector from the direction given by the helical magnetic anisotropy and this results in the hysteresis observed in the left GMI peaks (Fig. 5). The asymmetry becomes evident, when $i_{\mathrm{dc}}>i_{\mathrm{ac}}$, i.e., the microwire is strongly asymmetrically magnetized in one circular direction only. Consequently the hysteresis vanishes, the peak position $\pm H_{\mathrm{m}}$ increases and the asymmetric GMI magnitude decreases by about $40 \%$. 


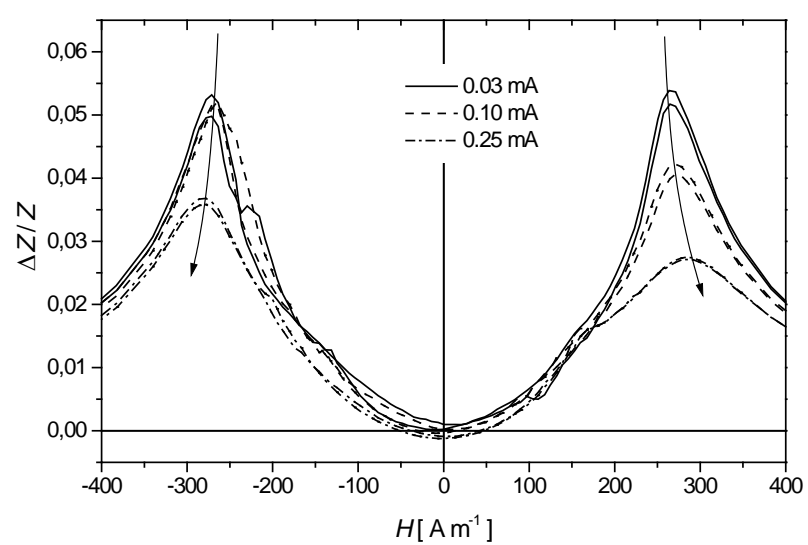

Fig. 5 The asymmetric GMI effect in $\mathrm{Co}_{70.5} \mathrm{Fe}_{4.5} \mathrm{Si}_{15} \mathrm{~B}_{10}$ microwire during magnetization rotation measured at frequency of $1 \mathrm{MHz}$ with AC current (circular magnetic field) amplitude $i_{\mathrm{ac}}$ of $0.1 \mathrm{~mA}$ and applied DC bias current $i_{\mathrm{dc}}$ from 0.03 to $0.25 \mathrm{~mA}$

\section{CONCLUSION}

The maximum GMI ratio $(\Delta Z / Z)_{\max }$ dependence on the amplitude $i_{\mathrm{ac}}$ (or circular field $H_{\phi}=i_{\mathrm{ac}} / 2 \pi a$ ) was analyzed by means of the helical anisotropy $\left(0<\alpha<90^{\circ}\right)$.

It can be concluded that

- if the reversible magnetization rotation occurs, the maximum GMI ratio $(\Delta Z / Z)_{\max }$ increases with amplitude $i_{\mathrm{ac}} \leq 1 \mathrm{~mA}$ (or circular field $H_{\phi} \leq 22.7 \mathrm{Am}^{-1}$ ),

- if the irreversible rotation of magnetization starts at $i_{\mathrm{ac}}$ $>1 \mathrm{~mA}$ (or circular field $H_{\phi}>22.7 \mathrm{Am}^{-1}$ ), the hysteretic behaviour is observed in the measured GMI dependences.

When the microwire is magnetized in one circular direction with the additional dc bias current $i_{\mathrm{dc}}$, the magnetization vector diverts asymmetrically from the direction given by the helical magnetic anisotropy $\left(0<\alpha<90^{\circ}\right)$ existing in the glass-coated ferromagnetic amorphous $\mathrm{CoFeSiB}$ microwire and this results in the asymmetry as well as in the hysteresis observed in the GMI dependences.

\section{ACKNOWLEDGMENT}

The "We support research activities in Slovakia"" project is co-financed from EU funds. This paper was developed as part of the project named "Centre of Excellence for Integrated Research \& Exploitation of Advanced Materials and Technologies in Automotive Electronics”, ITMS 26220120055.

\section{REFERENCES}

[1] VÁZQUEZ, M.: Soft magnetic wires, Physica B, vol. 299, p. 302-313, 2001.

[2] PANINA, L. V. - MOHRI, K. - UCHIYAMA, T NODA, M.: Giant Magneto-impedance in Co-Rich Amorphous Wires and Films, IEEE Transaction on Magnetics, vol. 31, no. 2, p. 1249-1260, 1995.

[3] KNOBEL, M. - VÁZQUEZ, M. - KRAUS, L.: Handbook of Magnetic Materials: chapter 5 Giant Magnetoimpedance (Ed. by K. H. J. Buschow), Vol. 15, Elsevier Science B.V., p. 497-563, 2003.

[4] CHIKAZUMI, S.: Physics of Magnetism: chapter 14.1 Irreversible Rotation Magnetization, John Wiley \& Sons, New York, p. 281-5, 1964.

[5] MACHADO, F. L. A. - REZENDE, S. M.: Journal of Applied Physics, Vol. 79, p. 6558-60, 1996.

[6] KRAVČÁK, J. - VARGA, R.: Asymmetric magneto-impedance in $\mathrm{CoFeSiB}$ amorphous microwires, Acta Physica Polonica A, Vol. 118, p. 762-3, Nov. 2010.

[7] USOV, N. A. - ANTONOV, A. S. - LAGARKOV, A. N.: Theory of giant magneto-impedance effect in amorphous wires with different types of magnetic anisotropy, Journal of Magnetism and Magnetic Materials, Vol. 185, p. 159-173, 1998.

Received November 25, 2012 , accepted February 9, 2013

\section{BIOGRAPHY}

Jozef Kravčák was born on 17.03.1972. In 1995 he graduated (MSc) from the Department of Physics of Condensed Matter of the Faculty of Science at P. J. Šafárik University in Košice. He defended his $\mathrm{PhD}$ thesis "Directional ordering in ferromagnetic alloys" in 2004. Since 1997 he is working as assistant professor at the Department of Physics of the Faculty of Electrical Engineering and Informatics at Technical University in Košice. His scientific research is focused on dynamical magnetic processes in amorphous magnetic materials. 